\title{
Insect Epicuticular Grease Visualised by Scanning Probe Microscopy
}

Stanislav Gorb, Dagmar Voigt, Henrik Peisker Evolutionary Biomaterials Group, Department of Thin-Films and Biological Systems, Max-Planck Institute for Metals Research, Stuttgart, Germany, email:s.gorb@mf.mpg.de

\section{Epicuticular grease}

The covering wings (elytra) in the Colorado potato beetle Leptinotarsa decemlineata appear shiny, smooth, water-repellent, and slightly slippery, (Fig. 1). These properties are due to the presence of epicuticle, the outermost layer of the insect integument, covered by a wax-like lipid surface layer called grease. Surface waxes have been previously reported in a variety of conditions, from liquid viscous coatings to crystalline structures in form of plates, rods and filaments from many insects (adults and larvae) and arachnids (Hadley, 1981a,b). Beament (1945) and Wigglesworth (1945) considered an outer thin layer of solid wax, whereas Lewis (1962) rather assumed an oil film on the epicuticle surface to be widespread throughout insects. According to Noble-Nesbitt

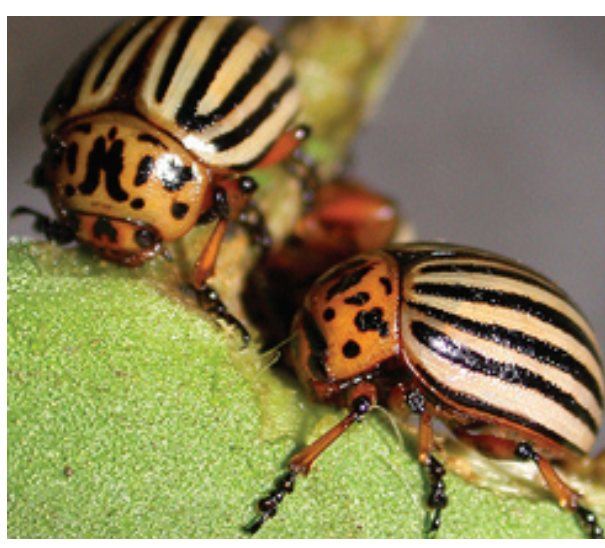

Fig 1. Colorado potato beetle Leptinotarsa decemlineata. (1992), epicuticular lipids are found to be either waxes or mobile greases at ambient temperatures. Outermost greasy layers on the epicuticle have been reported in cockroaches (Beament, 1945; 1958; Gilby and Cox, 1963), beetles (Richards, 1951), flies (Wolfe, 1954), true bugs (Wigglesworth, 1933), crickets (Hendricks and Hadley, 1983), locusts (Vötsch et al., 2002), ticks (Lees and Beament, 1948), and spiders (Hadley, 1981a; McConney et al., 2007).

The thickness of the superficial greasy wax layer covering epicuticle is of molecular dimensions (Beament, 1955) varying from less than a few $\mathrm{nm}$ to several $\mu \mathrm{m}$ (Hendricks and Hadley, 1983). The bulk of lipid on an insect's surface forms a layer of 0.1-1.0 $\mu \mathrm{m}$ thickness, probably in less well oriented layers permeating the cement layer of the epicuticle (Locke, 1964).

The greasy material on the cuticle surface is thought to play a fundamental role in preventing water loss (Ramsay, 1935; Neville, 1975). Furthermore, cuticular lipids may serve as chemical cues used by insects for signalling in olfactory communication (Espelie et al., 1991).

Detailed information on layer thickness, release, distribution, and physical properties of the grease is required. Previous morphological and ultrastructural studies using conventional electron microscopy methods (SEM, TEM) resulted in the removal of surface films, because samples were either dried and/or washed

in organic solvents, such as ethanol, acetone, and propylene oxide, according to conventional preparation procedures.

The present study was undertaken to characterize thickness of the epicuticular grease layer and its adhesive properties in beetle elytra. For this purpose, atomic force microscopy (AFM) was applied, which has been previously demonstrated to be an excellent approach for geometrical characterisation of insect and spider cuticles and for estimation of their adhesive properties on a local scale (Scherge and Gorb, 2001; Langer et al., 2004; McConney et al., 2007).

\section{Insects and sample preparation}

Beetle, Leptinotarsa decemlineata Say (Coleoptera, Chrysomelidae) (Fig. 2A), were taken from a stock culture $\left(25^{\circ} \mathrm{C}\right.$, $60 \% \mathrm{RH}, 16 \mathrm{~h}$ photoperiod) at Bayer Cropscience AG (Monheim, Germany). Elytra were removed from anaesthetized live females using micro-scissors and tweezers and immediately mounted on a glass slide using double-sided adhesive tape. In all, elytra of five female beetle individuals were studied.

\section{Scanning probe microscopy}

We used a NanoWizard atomic force microscope (JPK Instruments AG, Berlin, Germany) mounted on an inverted microscope Zeiss Axiovert 135 (Carl Zeiss MicroImaging GmbH, Göttingen, Germany). NanoWizard image acquisition software 3.1.13 (JPK Instruments AG, Berlin, Germany) was used to obtain AFM images and NanoWizard image processing software 3.1.6 was used to process images and calculate all necessary geometrical parameters of the surface. The most common operating modes in the AFM are tapping mode and contact mode. The latter provides a better resolution, whereas, in the tapping mode, both the normal force and shear force applied to the sample are minimized. Visualization of the epicuticular grease in both modes provided us with complementary information. The force applied to the surface, was set to a minimum to ensure the integrity of the sample in both operating modes.

Tapping mode. Scans were carried out in air at a scan rate of $1 \mathrm{~Hz}$ and a resolution of $512 \times 512$ pixels using standard, noncontact, high frequency cantilevers with reflex coating (NSTNCHF-R, Nascatec Technologies GmbH, Stuttgart, Germany). AFM images were taken from four pieces of the same elytron.

Contact mode. In contrast to the tapping mode, the contact mode always exerts a much larger lateral force, making it more difficult to properly visualize sensitive surfaces like insect epicuticular grease. However, load force must be sufficient to properly resolve the surface topography. The optimal balance between these two parameters was determined by scanning the same surface repeatedly while optimising the setpoint, scan rate and gains of the feedback loop. Scans with a size of $20 \times 20 \mu$ m were obtained at $1 \mathrm{~Hz}$ line rate and a resolution of $512 \times 512$ pixels using standard contact mode cantilevers (NST-CM, Nascatec Technologies GmbH, Stuttgart, Germany).

\section{Cryo-SEM}

Cryo-SEM allows observation of fresh specimens and provides a unique possibility to confirm our data obtained from AFM scans. Female elytra surfaces were analysed using a cryo-SEM Hitachi S 4800 (Hitachi High-Technologies Corp., Tokyo, Japan) equipped with a Gatan ALTO 2500 cryo-preparation system 



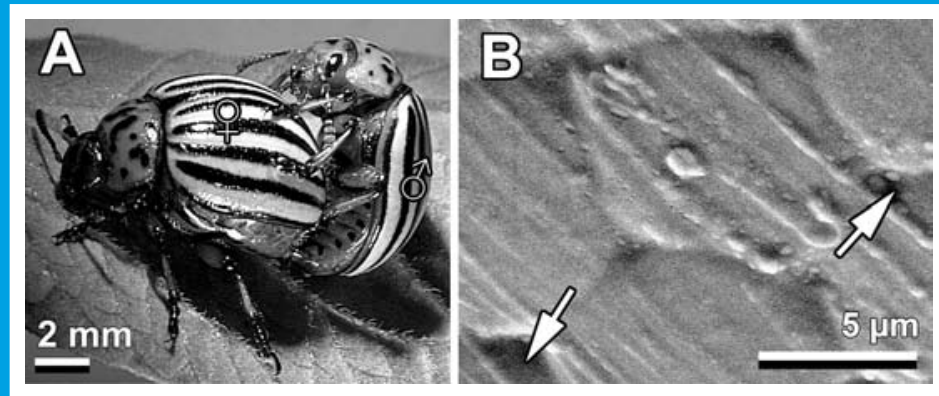

Fig. 2. A. Leptinotarsa decemlineata. B. Cryo SEM micrograph of the fresh female elytra surface. Small pores (arrows) and single cells of the hexagonal surface pattern. Grease smeared over the surface is visible.

(Gatan Inc., Abingdon, UK). Previously, this method has been successfully applied for visualization of tarsal adhesive fluid in flies (Gorb, 2006). Pieces of elytra from live, anaesthetized beetles were cut off with a fine knife, mounted on metal holders, frozen on a cryo-stage at $-140^{\circ} \mathrm{C}$, sputter-coated with gold-palladium $(3 \mathrm{~nm})$ in the preparation chamber, and examined in the SEM at $-120^{\circ} \mathrm{C}$ and an accelerating voltage of $5 \mathrm{kV}$.

\section{Grease visualization}

Cryo-SEM images of fresh, shock-frozen elytra demonstrated pores distributed over the surface (Fig. 2B). The pores observed are presumably responsible for delivering secretory substances to the cuticle surface. At higher magnification, an amorphous layer smeared over the surface can be detected (Fig. 2B). This layer often covers small pores and gaps between hexagons and thus makes the surface uniformly smooth.

The gathered AFM tapping mode height images of fresh samples provided 3D information about hexagonal cells at the nanoscale (Fig. 3A, B). However, no grease was visible. In order to obtain a more detailed view of fresh surfaces, phase images were taken and compared to height images (Fig. 3C). Height images alone did not allow a definition of the grease distribution over
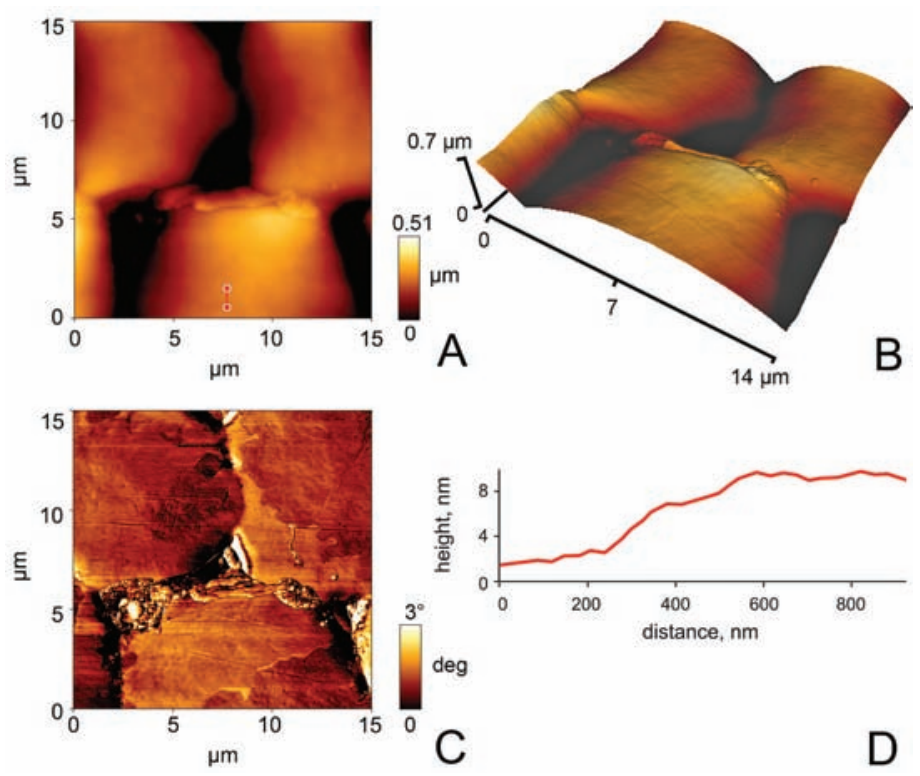

Fig. 3. AFM tapping mode images of a fresh female elytron of Leptinotarsa decemlineata. A. Height image. B. Phase image of $A$ indicating differences in surface properties/geometry by the use of phase shift visualization. Grease distribution (darker sites) is clearly visible. C. $3 D$ phase image showing the grease boundary. D. Profile diagram of the height image representing a cross section of the grease boundary along the line indicated between two dots in A. the surface, whereas phase images showed distinct differences in material properties and/or geometry indicated by a phase shift. The grease often accumulated in the vicinity of small pores, within the pores themselves, or in the regions between cells. The grease layer was about $8 \mathrm{~nm}$ thick, shown by the profile crossing the boundary between a grease patch and the surface of the epicuticle (Fig. 3D).

Topographical images of the fresh elytra, obtained in the contact mode, showed a heavy, smeary, striped appearance especially in the proximity of cuticle pores (Fig. 4). The visualization of surface fine structure was disturbed in the vicinity of the smear. An attempt to decrease the force applied to the sample surface resulted in almost non-interpretable images of surface topography.

This case study demonstrates the application of the AFM for visualization of the epicuticular grease in insects. The location and thickness of the grease was demonstrated, showing its non-uniform distribution over the elytra surface. The grease residues also remain on the top of the hexagonal cells in the form of patches, whereas the rest runs down the curvature and

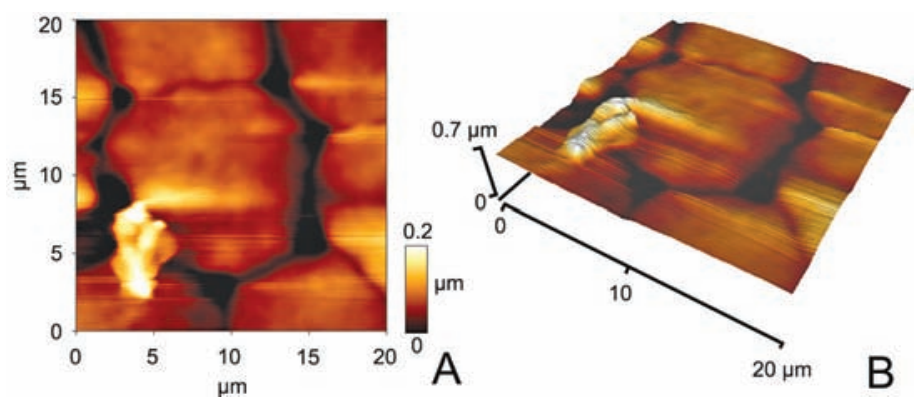

Fig. 4. AFM contact mode scans of a fresh female elytron of Leptinotarsa decemlineata. A. Height image. B. 3D image. Images show smearing artefacts, presumably caused by pushing cuticle grease with the cantilever tip.

accumulates within the pores. Also, grease accumulation between the cells is clearly visible.

The data, obtained with the AFM, were partly confirmed with the use of Cryo-SEM, which supported the presence and geometry of the grease layer. These data, providing an important extension to the previous data of chemical analyses, were obtained for the first time with both microscopy methods.

The combined use of both scanning modes (tapping and contact) shows the semi-fluid nature of the grease layer: the contact mode resulted in smeared images of the elytron surface, corresponding to fluid properties, whereas the tapping mode provided clear images of the surface, corresponding to solid properties.

Phase image, used in this study for visualization of the grease, has demonstrated very good results in combination with height images obtained in the tapping mode. Phase images aided in localisation of places on the smooth epicuticle covered with the grease. In spite of uncertainty of the origin of such a phase contrast (caused either by (1) surface geometry, (2) differences in local material properties or (3) both), it seems to be a powerful tool for detection and visualization of submicron-thick layers on the smooth surface of biological objects.

\section{Conclusion}

This study demonstrates three different AFM methods (contact mode, tapping mode, and phase contrast modes) for 
visualization of cuticle grease in living insect cuticle. Cryo-SEM was applied as a control method to confirm data obtained with the AFM. The grease layer was about $8 \mathrm{~nm}$ thick. Grease accumulation was observed between the hexagonally-shaped cells of the cuticle and within the pores.

\section{Acknowledgements}

Peter Meisner, Bayer Cropscience AG, Research Insecticides/ Insecticide Biology (Monheim, Germany) kindly provided the insects. Valuable discussions with Michael Varenberg (Technion, Haifa, Israel) on contact mechanics and tribology are greatly acknowledged. Cristian Löbbe (JPK, Berlin, Germany) helped with the data processing. Victoria Kastner (Tübingen, Germany) provided linguistic corrections on an early draft of the manuscript. This study was partly funded by the Federal Ministry of Education and Research, Germany (project InspiRat 01RI0633D).

\section{References}

Beament JWL (1945) The cuticular lipoids of insects. LExp Biol 21:115-131

Beament JWL (1955) Wax secretion in the cockroach. J Exp Biol 32:514-538

Beament JWL (1958) The effect of temperature on the waterproofing mechanism of an insect. LExp Biol 35:494-519

Espelie KE, Bernays EA, Brown JJ (1991) Plant and insect cuticular lipids serve as behavioural cues for insects. Arch Ins Biochem Physiol 17:223-233

Gilby AR, Cox ME (1963) The cuticular lipids of the cockroach Periplaneta americana (L.). I Insect Physiol 9:671-681

Gorb SN (2006) Fly microdroplets viewed big: a Cryo-SEM approach. Microscopy Today N9:38-39

Hadley NF (1981a) Fine structure of the cuticle of the black widow spider with reference to surface lipids. Tissue and Cell 13:805-817

Hadley NF (1981b) Cuticular lipids of terrestrial plants and arthropods: A comparison of their structure, composition, and waterproofing function. Biol Rev 56:23-47
Hendricks GM, Hadley NF (1983) Structure of the cuticle of the common house cricket with reference to the location of lipids. Tissue \& Cell 15:761-779

Langer MG, Ruppersberg JP, Gorb S (2004) Adhesion forces measured at the level of a terminal plate of the fly's seta. Proc R Soc Lond B 271:2209-2215

Lees AD and Beament JWL (1948) An egg-waxing organ in ticks. Quart J Micr Sci 89:291-332

Lewis CT (1962) Diffusion of oil films over insects. Nature 183:904

Locke M (1964) The structure and formation of the integument in insects. In: Rockstein M (ed.) The Physiology of Insecta. Academic Press, New York, 123-213

McConney ME, Schaber CF, Julian MD, Barth FG, Tsukruk VV (2007) Viscoelastic nanoscale properties of cuticle contribute to the high-pass properties of spider vibration receptor (Cupiennius salei Keys). I R Soc Interface 4:1135-1143

Neville AC (1975) Biology of the arthropod cuticle. Springer, Berlin

Noble-Nesbitt J (1992) Cuticular permeability and its control. In: Binnington K, Retnakaran A (eds.) Physiology of the insect epidermis. CSIRO Australia:252-283

Ramsay JA (1935) The evaporation of water from the cockroach. J Exp Biol $12: 373$

Richards AG (1951) The integument of arthropods. The chemical components and their properties, the anatomy and development, and the permeability. University of Minnesota Press, Minneapolis

Scherge M, Gorb SN (2001) Biological micro- and nanotribology. Springer, Berlin Heidelberg, New York

Vötsch W, Nicholson G, Müller R, Stierhof Y-D, Gorb S, Schwarz U (2002) Chemical composition of the attachment pad secretion of the locust Locusta migratoria. Insect Biochem Mol Biol 32:1605-1613

Wigglesworth VB (1933) The physiology of the cuticle and of ecdysis in Rhodnius prolixus with special reference to the function of the oenocytes and of the dermal glands. Quart J Micr Sci 76:269

Wigglesworth VB (1945) The insect cuticle. Biol Rev 23:408-451

Wolfe LS (1954) Studies of the development of the imaginal cuticle of Calliphora erythrocephala. J Cell Sci s3-95:67-78

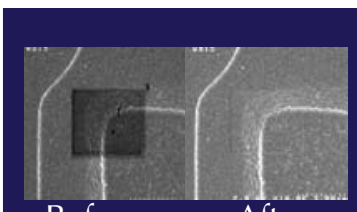

Intelligent Design

A new design for our

controller and RF section greatly increases

Remote Plasma Cleaning from XEI Scientific, Inc

Before After removes hydrocarbon contamination for SEMs and FIBs cleaning efficiency and reliability
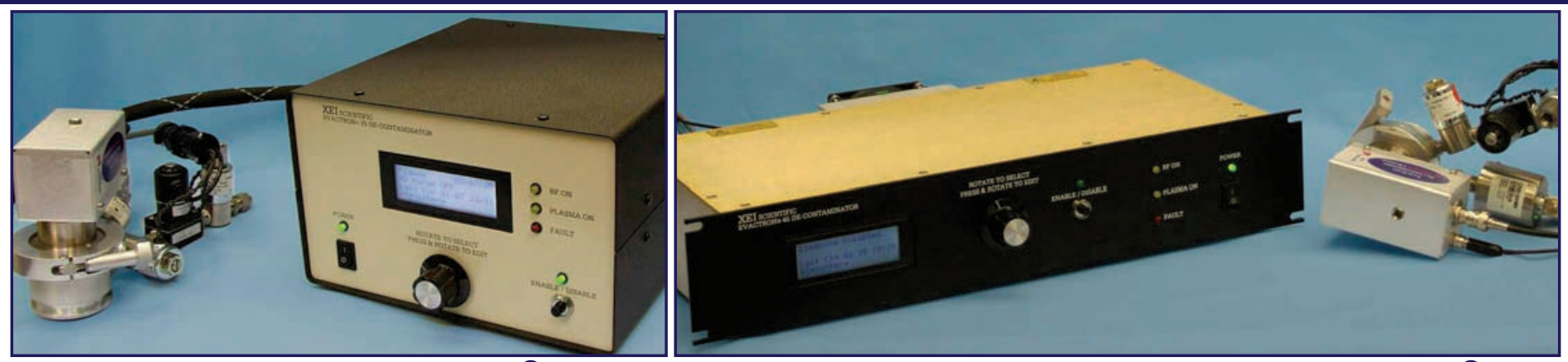

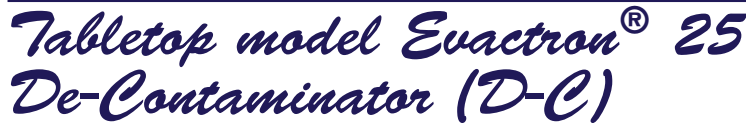

Evactron ${ }^{\circledR}$ D-C gently cleans your chamber using oxygen radicals to remove atmospheric molecular contamination, oils and other hydrocarbons through chemical ashing. Our latest Evactron ${ }^{\circledR}$ models for SEM and FIB chamber cleaning have advanced microprocessor control, allowing the operator to pre-set cleaning time, pressure and power. Our new matching network maximizes oxygen radical production in our low power, remote RF plasma. New plasma detection logic insures plasma ignition and oxygen radical production. An operational log keeps a record of your cleaning. All these features help you get clean chambers and great pictures!

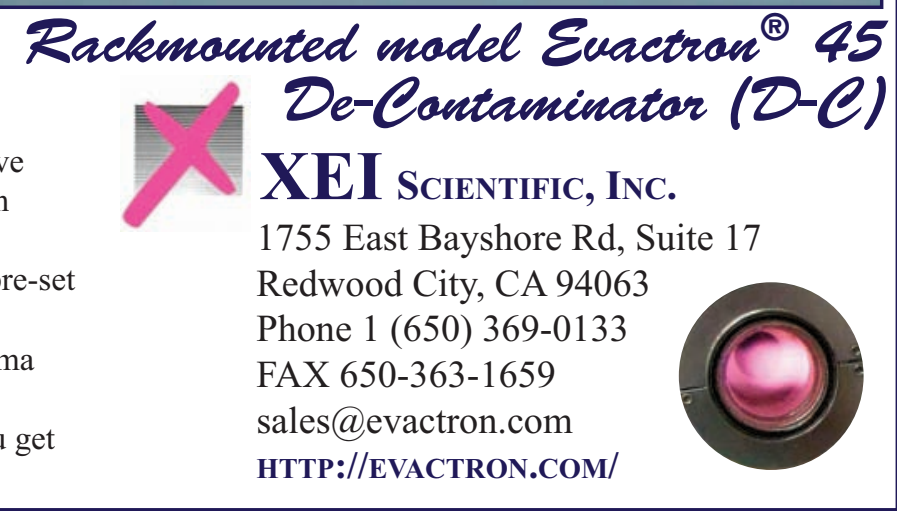

